\title{
A hybrid approach of neutrosophic sets and DEMATEL method for developing supplier selection criteria
}

\author{
Mohamed Abdel-Basset $^{1}$ - Gunasekaran Manogaran ${ }^{2}$ • Abduallah Gamal ${ }^{1}$. \\ Florentin Smarandache ${ }^{3}$
}

Received: 5 February 2018 / Accepted: 8 March 2018 / Published online: 27 March 2018

(C) Springer Science+Business Media, LLC, part of Springer Nature 2018

\begin{abstract}
For any organization, the selection of suppliers is a very important step to increase productivity and profitability. Any organization or company seeks to use the best methodology and the appropriate technology to achieve its strategies and objectives. The present study employs the neutrosophic set for decision making and evaluation method (DEMATEL) to analyze and determine the factors influencing the selection of SCM suppliers. DEMATEL is considered a proactive approach to improve performance and achieve competitive advantages. This study applies the neutrosophic set Theory to adjust general judgment, using a new scale to present each value. A case study implementing the proposed methodology is presented (i.e. selecting the best supplier for a distribution company). This research was designed by neutrosophic DEMATEL data collection survey of experts, interviewing professionals in management, procurement and production. The results analyzed in our research prove that quality is the most influential criterion in the selection of suppliers.
\end{abstract}

Keywords Supply chain management (SCM) - Supplier selection · Neutrosophic set · DEMATEL

Mohamed Abdel-Basset

analyst_mohamed@yahoo.com

Gunasekaran Manogaran gokulmecse@gmail.com

Abduallah Gamal

abdallagamalm@gmail.com

Florentin Smarandache

fsmarandache@gmail.com

1 Department of Operations Research, Faculty of Computers and Informatics, Zagazig University, Zagazig, Sharqiyah, Egypt

2 University of California, Davis, CA, USA

3 Mathematics \& Science Department, University of New Mexico, Gallup, NM 87301, USA 


\section{Introduction}

It cannot be denied that the success or the failure of any organization depend on how it chooses the appropriate supply chain management system and suppliers. Many organizations are currently seeking to contract many suppliers from around the world to create a collaborative commerce, and to increase trade, profitability and productivity. Experts are interested in purchasing and holding contracts with major suppliers, since the supplier selection is one of the most important functions of saving raw materials cost, of procurement management, and of increasing competitive advantage. The supply chain is an integral part of the new business management in the design of services from suppliers to customers. Supply chain management enables business participants to effectively combine products and services for a long-term relationship [1]. The effective coordination on information flows between enterprises, material, delivery, product, payment and trading partners can be defined extensively as supply chain management [2]. The economic environment forces organizations and collective institutions to seek competitive alternatives to meet the needs of customers and market. Organizations must have better production technology for internal and external competitiveness. Companies are an important part of the process of increasing the supply chain. Projects seeking to increase the production and compete in the international market must manage the supply chain in a highly effective way, and the suppliers selection is considered a key point of the process [3]. The process of integrating all activities in order to create satisfied customers is called supply chain management, and it is applied by the best companies around the world to control the flow of information, services and materials [4]. Supply chain management improves the competitive position of a company. Companies are always striving to maintain their competitiveness by developing issues such as improved model analysis, road planning, pregnancy planning, or supply chain management. Usually, the managers focus on organizing processes within the company to maximize profits, but the supply chain management seeks to link internal processes and decisions with external enterprise partners to improve and create competitiveness [5]. In recent years, supply chain management has attracted increasing awareness in academic publications. Supply chain management has been used to promote efficiency of the value chain on a wide range of products, services and other manufactured materials. Disagreement may occur in the process of selecting criteria. Many studies have tried to help managers and decision makers in any organization to take a relevant decision in selecting the best criterion suiting their organizations.

The process of supplier evaluation, appraisal, evaluation and contracting is called supplier selection [6]. There are some distinguishing features among suppliers, such as manufacturing procedures, technology, geographical location and larger processes that adopt better suppliers in pursuit of competitiveness [7]. Many researchers are tempted in displaying performance to make the supply chain more and more efficient [8], and consequently an intuitionistic fuzzy sets DEMATEL method was proposed to analyze the influential criteria practices, suggesting that empirical studies should be conducted as future research [9]. DEMATEL, an extended technique for formulating and analyzing influential relationships among difficult criteria, has been extensively used to extract the texture of a complex problem. The current literature review indicates that most papers used traditional intuitionistic fuzzy set to level the ambiguity of experts judgments and opinions (Fig. 1).

Fuzzy set focuses only on membership function and it does not take into account the non-membership and indeterminacy, so it fails to deal with uncertainty and indeterminacy existing in the real world. To overcome the drawbacks of fuzzy set, we integrated DEMATEL method in neutrosophic environment. The neutrosophic set is an extent or generalization of 


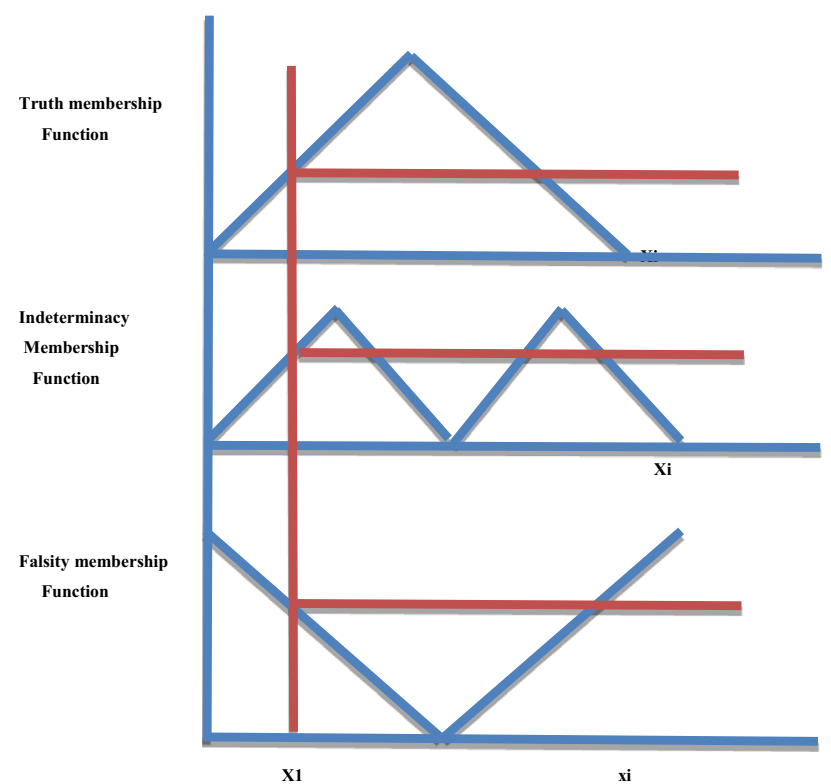

Fig. 1 Neutrosophication process [16]

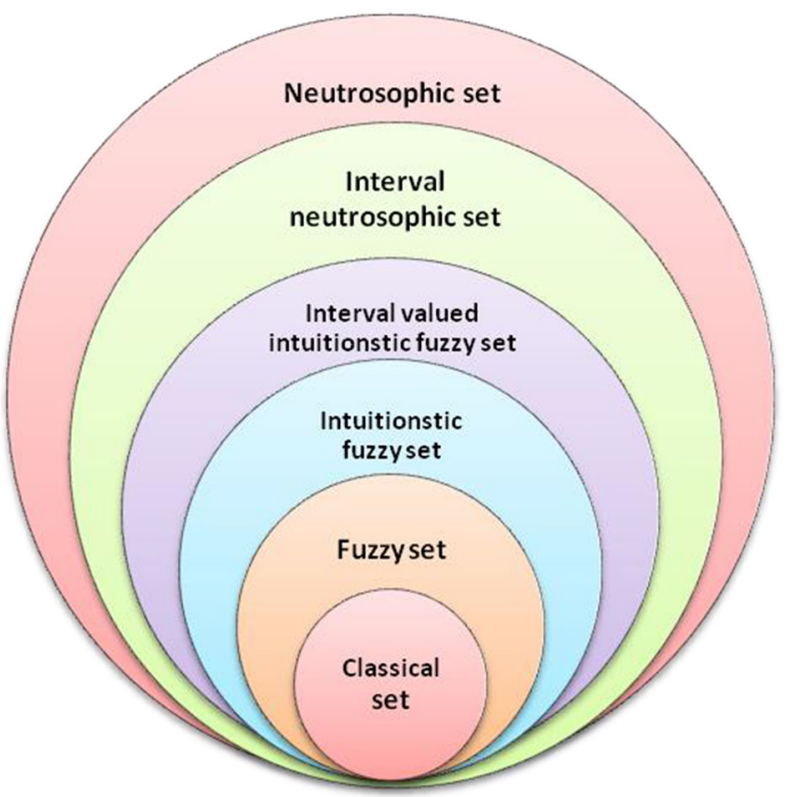

Fig. 2 From classical sets to neutrosophic sets

the intuitionistic fuzzy set. It represents real world problems effectively and efficiently by considering all aspects of a decision situations (i.e. truthiness, indeterminacy and falsity) [10, 11], as shown in Fig. 2. 
Neutrosophy was introduced by Smarandache $[12,13]$ as a branch of philosophy that studies the origin and scope of neutralities. Neutrosophy has been used in various applications to solve various problems as a critical path problem [14], obtaining PERT three times in project management [15]. Normally, the criteria have a degree of interactivity and related relationships. In such cases, it is very difficult for decision makers and experts to avoid interference between criteria and to obtain a specific goal. The main contributions of this research are:

- It introduces a new methodology by aggregating the neutrosophic set and DEMATEL method.

- It presents a case study showing how an organization increases its practices and activities according to specific criteria.

In this research, the DEMATEL method is used to develop mutual relationships and interdependencies. We present a causal diagram to describe relationships and their influence degrees on criteria.

It is important to evaluate the weakness and the strength of each criterion against another. One advantage of this method is showing the relationships and interdependence between features. Neutrosophic set theory is used in this research to express decision maker's preferences [17]. Neutrosophic sets (NSs) are an extension of the intuitionistic fuzzy sets (IFs), presenting more accurately the opinions and better interpreting the ambiguity, where the membership of a value or an element is defined as a number between 0 and 1, by resorting on a hesitation degree in IFs, whilst in NSs on an indeterminacy degree. Neutrosophic Set moves one step further by examining the membership of truth, the membership of indeterminacy and the non-membership of a member of a given set. Also, it is necessary to acquire experts opinions to evaluate influences. Neutrosophic Set has the following benefits:

- It introduces the indeterminacy degree that helps experts to express their opinions more accurately.

- It represents the extent of decision makers disagreements.

The proposed model also combines different interests of decision makers in one opinion in order to eliminate inconsistencies or to address the inconsistencies of expert judgments and improve consistency. A case study is solved to explain the model's suitability.

This research is organized as follows: Sect. 2 is a literature review that presents papers about DEMATEL for supplier selection. Section 3 illustrates the basic definitions of neutrosophic sets. Section 4 presents a methodology of the proposed model. Section 5 validates the model by solving a case study. Section 6 concludes the research and determines the future directions of the work.

\section{The related work}

In this section, we present some supplier selection related work. The two important stages in supply chain management, which considers all the activities from the purchasing of raw material to the final delivery of the product, are the supplier's selection and the evaluation. The supplier selection problem requires high accuracy methods of multiple criteria decision making for solving it. According to the literature reviews, many researchers proposed methods based on DEMATEL. Chang et al. [18] applied DEMATEL with fuzzy to evaluate and select the best supplier and to improve performance with respect to organizational factors and strategic performances, which included ten evaluating criteria. Dey et al. [19] applied 
DEMATEL to establish a long-term relationship with a company and its suppliers, with respect to their criteria. Hsu et al. [20] explored and used DEMATEL for decision making within the green supply chain, and focused on the components of green supply chain management and how they serve as a foundation for the decision framework and for recognizing the influential criterion of carbon footprint in environment. Lin [21] used DEMATEL to enhance environment performance, which is shaped by criteria as green purchasing, green design and product recovery practices. Dalalah et al. [22] employed DEMATEL for a supplier selection problem, implementing and applying it on an industrial case for the selection of cans suppliers at a factory in Amman, with respect to various supplier evaluation criteria. Govindan et al. [23] developed and used intuitionistic fuzzy with DEMATEL for decision making within the green supply chain, and focused on the components of green supply chain management to handle the causal relationships between GSCM practices and performances.

In this study, we aim to select the best supplier with respect to the various criteria using DEMATEL in neutrosophic environment. The selection of supplier problem is still challenging, and selecting the right supplier becomes a critical activity within a company, consequently affecting its efficiency and profitability. Due to its strategic importance, important research is being done to cope with the supplier evaluation and selection problem.

\section{Neutrosophic sets}

In this section, we give definitions involving neutrosophic sets, single valued neutrosophic sets, trapezoidal neutrosophic numbers, and operations on trapezoidal neutrosophic numbers.

Definition 1 [24] Let $X$ be a space of points and $x \in X$. A neutrosophic set in $X$ is defined by a truth-membership function $T_{A}(x)$, an indeterminacy-membership function $I_{A}(x)$ and a falsitymembership function $F_{A}(x), T_{A}(x), I_{A}(x)$ and $F_{A}(x)$ are real standard or real nonstandard subsets of $]-0,1+\left[\right.$. That is $\left.T_{A}(x): X \rightarrow\right]-0,1+\left[, I_{A}(x): X \rightarrow\right]-0,1+\left[\right.$ and $\left.F_{A}(x): X \rightarrow\right]-0$, $1+\left[\right.$. There is no restriction on the sum of $T_{A}(x), I_{A}(x)$ and $F_{A}(x)$, so $0-\leq \sup (x)+\sup x$ $+\sup x \leq 3+$.

Definition 2 [16, 24-26] Let $X$ be an universe of discourse. A single valued neutrosophic set over $X$ is an object taking the form $=\left\{\left\langle x, T_{A}(x), I_{A}(x), F_{A}(x),\right\rangle: x \in X\right\}$, where $T_{A}(x): X$ $\rightarrow[0,1], I_{A}(x): X \rightarrow[0,1]$ and $F_{A}(x): X \rightarrow[0,1]$ with $0 \leq T_{A}(x)+I_{A}(x)+F_{A}(x) \leq 3$ for all $x \in X$. The intervals $T_{A}(x), I_{A}(x)$ and $F_{A}(x)$ represent the truth-membership degree, the indeterminacy-membership degree and the falsity membership degree of $x$ to , respectively. For convenience, a SVN number is represented by $=(a, \mathrm{~b}, \mathrm{c})$, where $a, b, c \in[0,1]$ and $a+b$ $+c \leq 3$.

Definition $3[27,28]$ Suppose $\alpha_{\tilde{a}}, \theta_{\tilde{a}}, \beta_{\widetilde{a}} \in[0,1]$ and $a_{1}, a_{2}, a_{3}, a_{4} \in \mathrm{R}$, where $a_{1} \leq a_{2} \leq a_{3}$ $\leq a_{4}$. Then, a single valued trapezoidal neutrosophic number $\tilde{a}=\left\langle\left(a_{1}, a_{2}, a_{3}, a_{4}\right) ; \alpha_{\tilde{a}}, \theta_{\tilde{a}}, \beta_{\tilde{a}}\right\rangle$ is a special neutrosophic set on the real line set $\mathrm{R}$, whose truth-membership, indeterminacymembership and falsity-membership functions are defined as:

$$
T_{\widetilde{a}}= \begin{cases}\alpha_{\widetilde{a}}\left(\frac{x-a_{1}}{a_{2}-a_{1}}\right) & \left(a_{1} \leq x \leq a_{2}\right) \\ \alpha_{\widetilde{a}} & \left(a_{2} \leq x \leq a_{3}\right) \\ \alpha_{\widetilde{a}}\left(\frac{a_{4}-x}{a_{4}-a_{3}}\right) & \left(a_{3} \leq x \leq a_{4}\right) \\ 0 & \text { otherwise }\end{cases}
$$




$$
\begin{gathered}
I_{\widetilde{a}}(x)= \begin{cases}\frac{\left(a_{2}-x+\theta_{\tilde{a}}\left(x-a_{1}\right)\right)}{\left(a_{2}-a_{1}\right)} & \left(a_{1} \leq x \leq a_{2}\right) \\
\alpha_{\tilde{a}} & \left(a_{2} \leq x \leq a_{3}\right) \\
\frac{\left(x-a_{3}+\theta_{\tilde{a}}(a 4-x)\right)}{\left(a_{4}-a_{3}\right)} & \left(a_{3} \leq x \leq a_{4}\right) \\
1 & \text { otherwise }\end{cases} \\
F_{\widetilde{a}}(x)=\left\{\begin{array}{ll}
\frac{\left(a_{2}-x+\beta_{\tilde{a}}\left(x-a_{1}\right)\right)}{\left(a_{2}-a_{1}\right)} & \left(a_{1} \leq x \leq a_{2}\right) \\
\alpha_{\tilde{a}} & \left(a_{2} \leq x \leq a_{3}\right) \\
\frac{\left(x-a_{3}+\beta_{\tilde{a}}(a 4-x)\right)}{\left(a_{4}-a_{3}\right)} & \left(a_{3} \leq x \leq a_{4}\right) \\
1 & \text { otherwise }
\end{array},\right.
\end{gathered}
$$

where $\alpha_{\widetilde{a}}, \theta_{\widetilde{a}}$ and $\beta_{\widetilde{a}}$ typify the maximum truth-membership degree, the minimum indeterminacy-membership degree and the minimum falsity-membership degree, respectively. A single valued trapezoidal neutrosophic number $\tilde{a}=\left\langle\left(a_{1}, a_{2}, a_{3}, a_{4}\right) ; \alpha_{\tilde{a}}, \theta_{\tilde{a}}, \beta_{\widetilde{a}}\right\rangle$ may express an ill-defined quantity of the range, which is approximately equal to the interval $\left[a_{2}\right.$, $\left.a_{3}\right]$.

Definition $4[16,28]$ Let $\tilde{a}=\left\langle\left(a_{1}, a_{2}, a_{3}, a_{4}\right) ; \alpha_{\tilde{a}}, \theta_{\tilde{a}}, \beta_{\widetilde{a}}\right\rangle$ and $\widetilde{b}=\left\langle\left(b_{1}, b_{2}, b_{3}, b_{4}\right) ; \alpha_{\tilde{a}}, \theta_{\tilde{a}}\right.$, $\left.\beta_{\widetilde{a}}\right\rangle$ be two single valued trapezoidal neutrosophic numbers, and $\Upsilon \neq 0$ be any real number. Then:

1. Addition of two trapezoidal neutrosophic numbers:

$$
\widetilde{a}+\widetilde{b}=\left\langle\left(a_{1}+b_{1}, a_{2}+b_{2}, a_{3}+b_{3}, a_{4}+b_{4}\right) ; \alpha_{\widetilde{a}} \wedge \alpha_{\widetilde{b}}, \theta_{\tilde{a}} \vee \theta_{\widetilde{b}}, \beta_{\widetilde{a}} \vee \beta_{\widetilde{b}}\right\rangle
$$

2. Subtraction of two trapezoidal neutrosophic numbers:

$$
\tilde{a}-\widetilde{b}=\left\langle\left(a_{1}-b_{4}, a_{2}-b_{3}, a_{3}-b_{2}, a_{4}-b_{1}\right) ; \alpha_{\widetilde{a}} \wedge \alpha_{\widetilde{b}}, \theta_{\widetilde{a}} \vee \theta_{\widetilde{b}}, \beta_{\widetilde{a}} \vee \beta_{\widetilde{b}}\right\rangle
$$

3. Inverse of trapezoidal neutrosophic numbers:

$$
\widetilde{\mathrm{a}}^{-1}=\left(\left(\frac{1}{a_{4}}, \frac{1}{a_{3}}, \frac{1}{a_{2}}, \frac{1}{a_{1}}\right) ; \alpha_{\tilde{a}}, \theta_{\tilde{a}}, \beta_{\widetilde{a}}\right) \text { where }(\tilde{a} \neq 0)
$$

4. Multiplication of trapezoidal neutrosophic numbers by constant value:

$$
\Upsilon \widetilde{a}= \begin{cases}\left\langle\left(\Upsilon a_{1}, \Upsilon a_{2}, \Upsilon a_{3}, \Upsilon a_{4}\right) ; \alpha_{\tilde{a}}, \theta_{\tilde{a}}, \beta_{\widetilde{a}}\right\rangle & \text { if }(\Upsilon>0) \\ \left\langle\left(\Upsilon a_{4}, \Upsilon a_{3}, \Upsilon a_{2}, \Upsilon a_{1}\right) ; \alpha_{\tilde{a}}, \theta_{\tilde{a}}, \beta_{\widetilde{a}}\right\rangle & \text { if }(\Upsilon<0)\end{cases}
$$

5. Division of two trapezoidal neutrosophic numbers:

$$
\underset{\widetilde{a}}{\widetilde{b}}=\left\{\begin{array}{l}
\left\langle\left(\frac{a_{1}}{b_{4}}, \frac{a_{2}}{b_{3}}, \frac{a_{3}}{b_{2}}, \frac{a_{4}}{b_{1}}\right) ; \alpha_{\widetilde{a}} \wedge \alpha_{\widetilde{b}}, \theta_{\widetilde{a}} \vee \theta_{\widetilde{b}}, \beta_{\widetilde{a}} \vee \beta_{\widetilde{b}}\right\rangle \text { if }\left(a_{4}>0, b_{4}>0\right) \\
\left\langle\left(\frac{a_{4}}{b_{4}}, \frac{a_{3}}{b_{3}}, \frac{a_{2}}{b_{2}}, \frac{a_{1}}{b_{1}}\right) ; \alpha_{\widetilde{a}} \wedge \alpha_{\widetilde{b}}, \theta_{\widetilde{a}} \vee \theta_{\widetilde{b}}, \beta_{\widetilde{a}} \vee \beta_{\widetilde{b}}\right\rangle \text { if }\left(a_{4}<0, b_{4}>0\right) \\
\left\langle\left(\frac{a_{4}}{b_{1}}, \frac{a_{3}}{b_{2}}, \frac{a_{2}}{b_{3}}, \frac{a_{1}}{b_{4}}\right) ; \alpha_{\widetilde{a}} \wedge \alpha_{\widetilde{b}}, \theta_{\widetilde{a}} \vee \theta_{\widetilde{b}}, \beta_{\widetilde{a}} \vee \beta_{\widetilde{b}}\right\rangle \text { if }\left(a_{4}<0, b_{4}<0\right)
\end{array}\right.
$$

6. Multiplication of trapezoidal neutrosophic numbers:

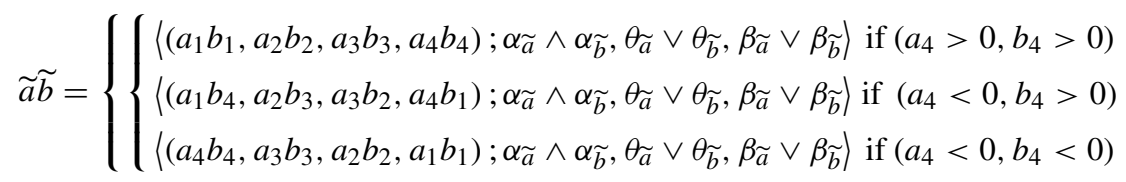




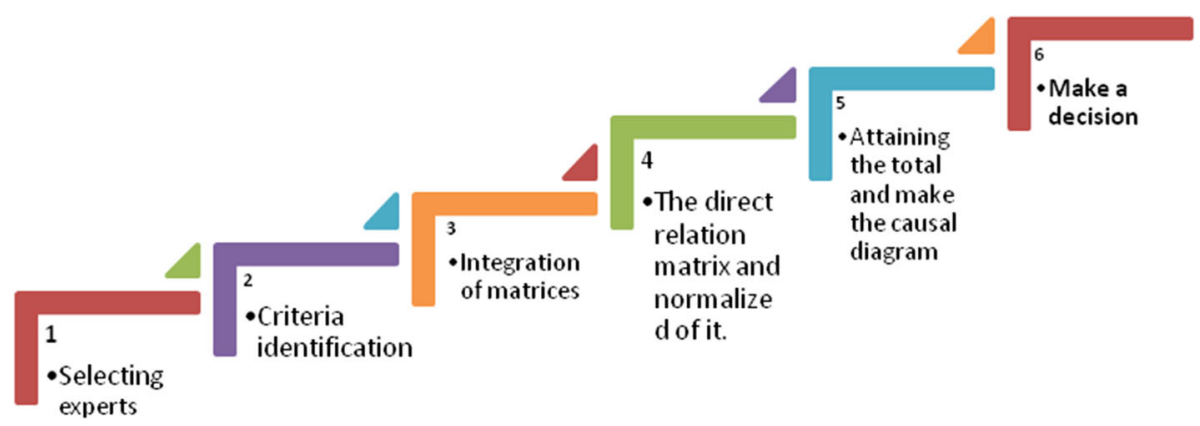

Fig. 3 The general neutrosophic DEMATEL framework

\section{Neutrosophic DEMATEL approach}

Atanassov [29] developed the intuitionistic fuzzy set theory. To overcome some of its limits, Smarandache [17] proposed the neutrosophic set theory. Neutrosophy handles vagueness and uncertainty, and attend the indeterminacy of values. Neutrosophy has some of advantage with DEMATEL:

- Neutrosophy provides the ability to present unknown information in our model using the indeterminacy degree, so the experts can present opinions about the unsure preferences.

- Neutrosophy depicts the disagreement of decision makers and experts.

- Neutrosophy heeds all aspects of decision making situations by considering truthiness, indeterminacy and falsity altogether.

DEMATEL is used to solve some complex and interrelated problems. In DEMATEL all criteria or factors fall into two categories: cause and effect.

In this section, we present the steps of the proposed model based on the neutrosophic DEMATEL analysis as shown in Fig. 3.

The procedures are explained as follows:

Step 1. Identifying decision goals: collecting relevant information presenting the problem.

1. Selection of experts and decision makers that have experience in the field.

2. Identifying the relevant criteria to the problem.

Step 2. Pairwise comparison matrices between relevant criteria.

1. Identify the criteria, Criteria $=(\mathrm{F} 1, \mathrm{~F} 2, \mathrm{~F} 3 \ldots \mathrm{Fm})$.

2. Experts make pairwise comparisons matrices between criteria.

a. Interpret each value for each criterion compared to other in a trapezoidal neutrosophic number $\left(l_{n m}, m_{n m l}, m_{n m}, u_{n m}\right)$.

b. Make comparisons between criteria by each expert as shown in Table 1 .

c. Focuses only on $(n-1)$ consensus judgments using a scale from 0 to $1[30,31]$.

3. Experts should determine the maximum truth-membership degree $(\alpha)$, the minimum indeterminacy-membership degree $(\beta)$ and the minimum falsity membership degree $(\theta)$ of single valued neutrosophic numbers as shown in Table 2.

4. Determine the crisp value of each opinion as shown in Table 3, using the following equations: 
Table 1 The pairwise comparison matrix between criteria

\begin{tabular}{lllll}
\hline Criteria & $\mathrm{F}_{1}$ & $\mathrm{~F}_{2}$ & $\ldots$ & $\mathrm{F}_{\mathrm{n}}$ \\
\hline $\mathrm{F}_{1}$ & $\left(l_{11}, m_{11 l}, m_{11 u}, u_{11}\right)$ & $\left(l_{11}, m_{11 l}, m_{11 u}, u_{11}\right)$ & $\ldots$ & $\left(l_{1 n}, m_{1 n l}, m_{1 n u}, u_{1 n}\right)$ \\
$\mathrm{F}_{2}$ & $\left(l_{21}, m_{21 l}, m_{21 u}, u_{21}\right)$ & $\left(l_{22}, m_{22 l}, m_{22 u}, u_{22}\right)$ & $\ldots$ & $\left(l_{2 n}, m_{2 n l}, m_{2 n u}, u_{2 n}\right)$ \\
$\ldots$ & $\ldots$ & $\ldots$ & $\ldots$ & $\ldots$ \\
$\mathrm{F}_{\mathrm{n}}$ & $\left(l_{n 1}, m_{n 1 l}, m_{n 1 u}, u_{n 1}\right)$ & $\left(l_{n 2}, m_{n 2 l}, m_{n 2 u}, u_{n 2}\right)$ & $\ldots$ & $\left(l_{n n}, m_{n n l}, m_{n n u}, u_{n n}\right)$
\end{tabular}

Table 2 The pairwise comparison matrix between criteria with the $\alpha, \beta$ and $\theta$ degree

\begin{tabular}{lllll}
\hline $\mathrm{C}$ & $\mathrm{F}_{1}$ & $\mathrm{~F}_{2}$ & $\ldots$ & $\mathrm{F}_{\mathrm{n}}$ \\
\hline $\mathrm{F}_{1}$ & $\left(l_{11}, m_{11 l}, m_{11 u}, u_{11} ; \alpha, \beta, \theta\right)$ & $\left(l_{11}, m_{11 l}, m_{11 u}, u_{11} ; \alpha, \beta, \theta\right)$ & $\ldots$ & $\left(l_{1 n}, m_{1 n l}, m_{1 n u}, u_{1 n} ; \alpha, \beta, \theta\right)$ \\
$\mathrm{F}_{2}$ & $\left(l_{21}, m_{21 l}, m_{21 u}, u_{21} ; \alpha, \beta, \theta\right)$ & $\left(l_{22}, m_{22 l}, m_{22 u}, u_{22} ; \alpha, \beta, \theta\right)$ & $\ldots$ & $\left(l_{2 n}, m_{2 n l}, m_{2 n u}, u_{2 n} ; \alpha, \beta, \theta\right)$ \\
$\ldots$ & $\ldots$ & $\ldots$ & $\ldots$ & $\ldots$ \\
$\mathrm{F}_{\mathrm{n}}$ & $\left(l_{n 1}, m_{n 1 l}, m_{n 1 u}, u_{n 1} ; \alpha, \beta, \theta\right)$ & $\left(l_{n 2}, m_{n 2 l}, m_{n 2 u}, u_{n 2} ; \alpha, \beta, \theta\right)$ & $\ldots$ & $\left(l_{n n}, m_{n n l}, m_{n n u}, u_{n n} ; \alpha, \beta, \theta\right)$ \\
\hline
\end{tabular}

Table 3 The crisp values of comparison matrix

Table 4 Integration of the average opinions of all experts

\begin{tabular}{lllll}
\hline $\mathrm{C}$ & $\mathrm{F}_{1}$ & $\mathrm{~F}_{2}$ & $\ldots$ & $\mathrm{F}_{\mathrm{n}}$ \\
\hline $\mathrm{F}_{1}$ & $C V_{11}$ & $C V_{21}$ & $\ldots$ & $C V_{m 1}$ \\
$\mathrm{~F}_{2}$ & $C V_{12}$ & $C V_{22}$ & $\ldots$ & $C V_{m 2}$ \\
$\ldots$ & $\ldots$ & $\ldots$ & $\ldots$ & $\ldots$ \\
$\mathrm{F}_{\mathrm{n}}$ & $C V_{1 n}$ & $C V_{2 n}$ & $\ldots$ & $C V_{m n}$ \\
\hline
\end{tabular}

\begin{tabular}{lllll}
\hline $\mathrm{C}$ & $\mathrm{F}_{1}$ & $\mathrm{~F}_{2}$ & $\ldots$ & $\mathrm{F}_{\mathrm{n}}$ \\
\hline $\mathrm{F}_{1}$ & $C V_{11}$ & $C V_{21}$ & $\ldots$ & $C V_{m 1}$ \\
$\mathrm{~F}_{2}$ & $C V_{12}$ & $C V_{22}$ & $\ldots$ & $C V_{m 2}$ \\
$\ldots$ & $\ldots$ & $\ldots$ & $\ldots$ & $\ldots$ \\
$\mathrm{F}_{\mathrm{n}}$ & $C V_{1 n}$ & $C V_{2 n}$ & $\ldots$ & $C V_{m n}$ \\
\hline
\end{tabular}

$$
\begin{aligned}
& \mathrm{S}\left(\widetilde{a}_{i j}\right)=\frac{1}{16}\left[a_{1}+b_{1}+c_{1}+d_{1}\right] \times\left(2+\alpha_{\widetilde{a}}-\theta_{\widetilde{a}}-\beta_{\widetilde{a}}\right) \\
& \mathrm{A}\left(\widetilde{\mathrm{a}}_{i j}\right)=\frac{1}{16}\left[a_{1}+b_{1}+c_{1}+d_{1}\right] \times\left(2+\alpha_{\tilde{a}}-\theta_{\tilde{a}}-\beta_{\widetilde{a}}\right)
\end{aligned}
$$

Step 3. Integration of matrices.

All opinions of experts need to be integrated into one matrix presenting the average opinions of all experts about each criterion, as shown in Table 4.

$$
C V_{11}=\frac{C V_{11 n 1}+C V_{11} n 2+C V_{11} n m}{n}
$$

where n, number of experts. We obtain the average for all values as in the following matrix.

Step 4. Generating the direct relation matrix. 
This matrix is obtained from previous step (3), i.e. the integrating of all averaged opinions of experts. An initial direct relation matrix $\mathrm{A}$ is a $\mathrm{n} \times \mathrm{n}$ matrix obtained by pairwise comparisons, $\mathrm{S}=\left[s_{i j}\right]_{n \times n}$. $S_{i j}$ denotes the degree to which the criterion i affects the criterion $\mathrm{j}$.

Step 5. Normalizing the direct relation matrix.

The normalized direct relation matrix can be obtained using the equation:

$$
\begin{gathered}
\mathrm{K}=\frac{1}{\operatorname{Max}_{1 \leq i \leq n} \sum_{j=1}^{n} a_{i j}} \\
\mathrm{~S}=\mathrm{K} \times \mathrm{A}
\end{gathered}
$$

Step 6. Attaining the total relation matrix.

This step requires use of the Matlab software. The total relation matrix is acquired using the formula (9) from the generalized direct relation matrix S. A total relation matrix (T), in which (I) denotes the identity matrix, is shown as follows:

$$
\mathrm{T}=\mathrm{S} \times(I-S)^{-1}
$$

Step 7. Obtaining the sum of rows and columns.

The sum of rows is denoted by (D), and the sum of columns is denoted by (R). Calculate $\mathrm{R}+\mathrm{D}$ and $\mathrm{R}-\mathrm{D}$.

Calculate $\mathrm{T}$, where $\mathrm{T}=\left[a_{i j}\right]_{n \times n}, \mathrm{i}, \mathrm{j}=1,2 \ldots \mathrm{n}$

$$
\begin{aligned}
& \mathrm{D}=\left[\sum_{i=1}^{n} a_{i j}\right]_{1 \times n}=\left[a_{j}\right]_{n \times 1} \\
& \mathrm{R}=\left[\sum_{j=1}^{n} a_{i j}\right]_{1 \times n}=\left[a_{j}\right]_{n \times 1}
\end{aligned}
$$

Step 8. Drawing cause and effect diagram

The causal diagram is obtained by the horizontal axes is presented by $(\mathrm{D}+\mathrm{R})$ and the vertical axes $(D-R)$ which is a degree of relation and it depicts the steps of proposed model in Fig. 4.

\section{The proposed methodology in a case study}

In this section, we describe the details of the proposed methodology of a hybrid approach of neutrosophic sets and DEMATEL method for developing supplier selection criteria. This section is divided into five subsections: (1) the case study, (2) the Neutrosophic DEMATEL questionnaire design, (3) the calculation process of the Neutrosophic DEMATEL Method, (4) the analysis of the evaluation criteria shown in Fig. 5.

\subsection{Case study}

Flopater Trading Company was established in 2003. The company specializes in supplying plastic pipe fittings, soon becoming one of the largest distributors for large companies in the production of PVC pipes and joints. The company started importing from abroad and took large contracts of polypropylene pipes and fittings produced by Cosmo Plast UAE. Cosmo Plast in the United Arab Emirates is one of the largest factories in the Gulf region, and the company has started to support this plant in projects and accreditation with consultants. 


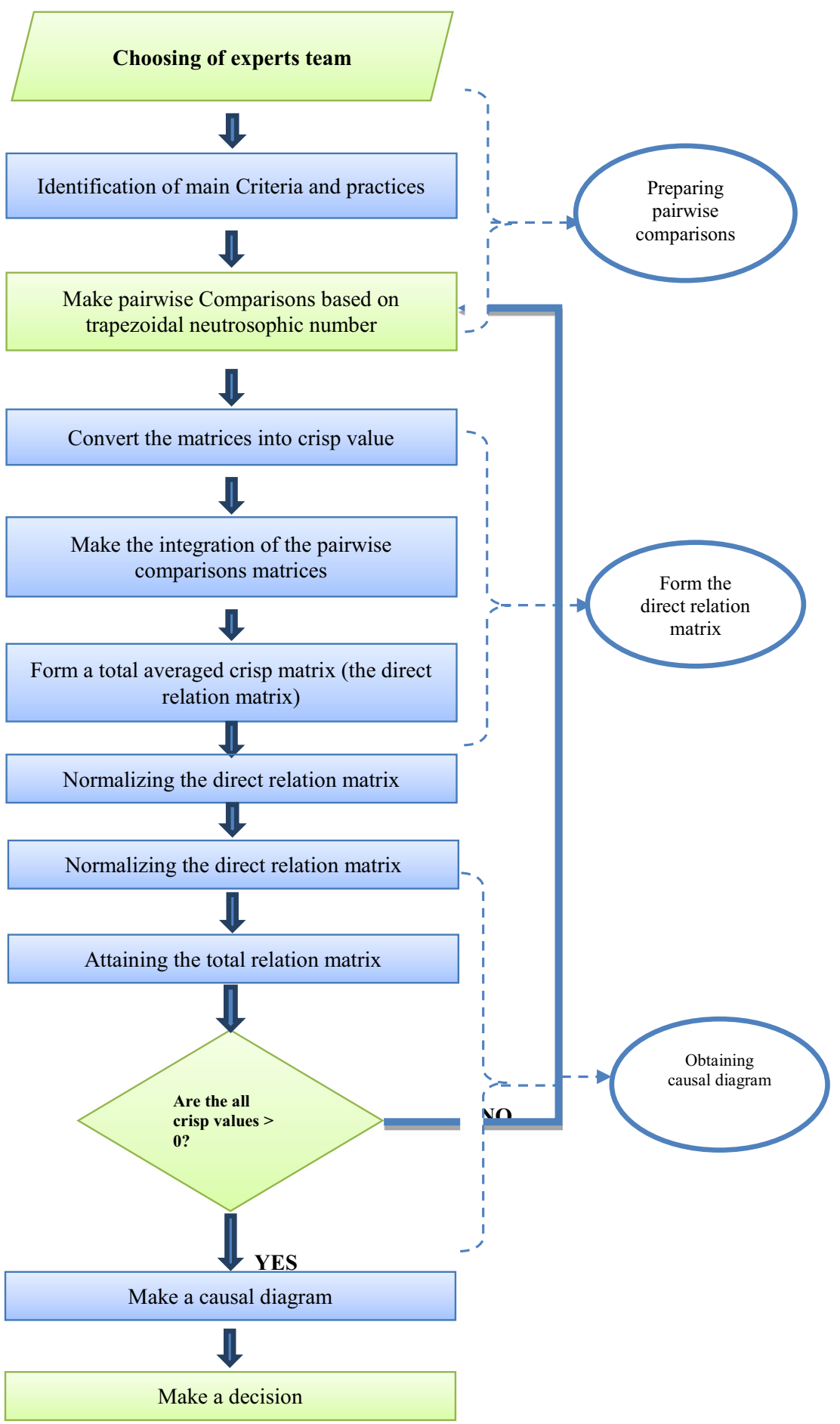

Fig. 4 Schematic diagram of DEMATEL in neutrosophic environment 


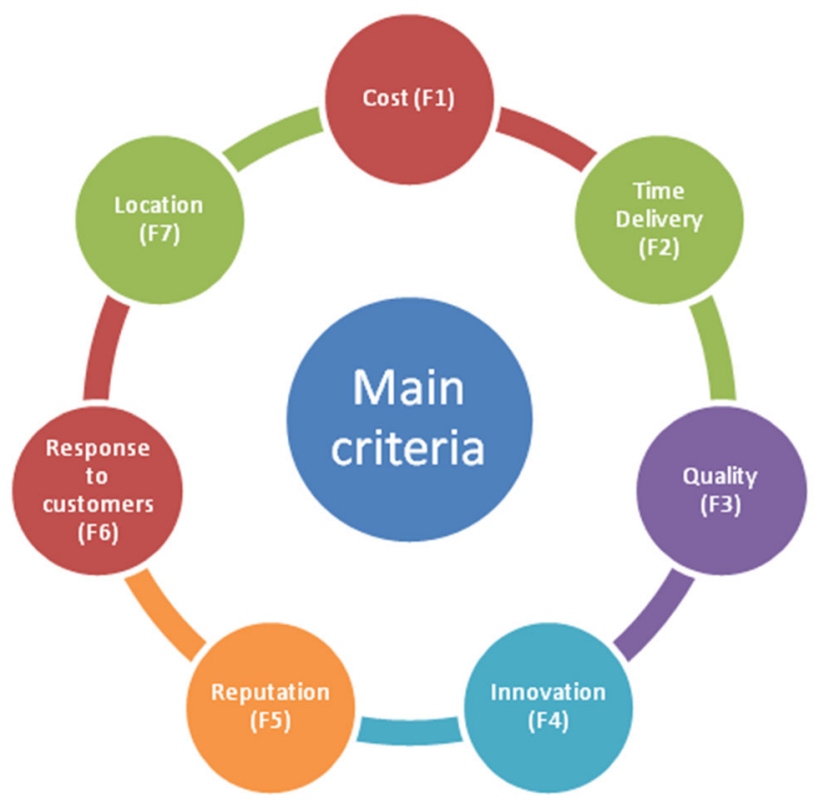

Fig. 5 Main criteria selected for evaluation

The company also imports chips produced by a major company in Turkey, Zir Kelipas, also providing full procurement to all contracting companies or contractors in projects, helping them to deliver all supplies to the place of the project or their stores. The company offers appropriate ways for payment, committing to always deliver on time. Since it intends to expand trade and increase the number of contracts with customers, one of the most important problems facing the company is the selection criteria of suppliers.

\subsection{Neutrosophic DEMATEL questionnaire design}

In this research, the design of questionnaire is structured as following: In the first part, we determine the selection criteria. Then, we need to understand each criterion, its definition and its importance in the evaluation of selecting supplier. We employed seven (7) evaluation criteria: (1) cost, (2) time delivery, (3) quality, (4) innovation, (5) reputation, (6) response to customers, (7) location. The influence of every criterion on selecting the best supplier gets evaluated by Neutrosophic DEMATEL method. In the second part, we perform pairwise comparisons matrices to evaluate each criterion based on points of views from experts, using the neutrosophic scale of 0,1 .

\subsection{The calculation process of Neutrosophic DEMATEL method}

For collecting data, we interviewed three professionals in the management of purchasing and setup of contracts. The three experts determined the most important evaluation criterion to be used. The criteria symbols in this research are as follows: Cost (F1), Time delivery (F2), Quality (F3), Innovation (F4), Reputation (F5), Response to customers (F6), Location (F7). The data collected from the three experts were analyzed by the Neutrosophic DEMATEL method. The steps that were conducted are the following: 
Step 1. Choosing the experts team

The first step of Neutrosophic DEMATEL method is selection of the best experts in the field of management purchasing and setup contracts. We selected three expert, to which we further refer as the first expert, the second expert, and the third expert.

Step 2. Identification of main criteria and practices

We sorted seven evaluation criteria as selected by the team of experts, namely: Cost (F1), Time delivery (F2), Quality (F3), Innovation (F4), Reputation (F5), Response to customers (F6), Location (F7).

Step 3. Performing pairwise comparisons matrices based on trapezoidal neutrosophic numbers.

1. Pairwise comparisons matrices to evaluate each feature or criterion against each other, as shown in Tables 5, 6, and 7.

2. Experts should determine the maximum truth membership degree $(\alpha)$, the minimum indeterminacy membership degree $(\theta)$ and the minimum falsity membership degree $(\beta)$ of single valued neutrosophic numbers, as shown in Tables 8, 9, 10.

3. Convert the matrices into crisp values, as shown in Tables 11, 12, 13.

Step 4. Integrating the matrices

We process the integration of the three matrices according to formula (6), where a diagonal is 0.5 . The initial direct-relation matrix $(\mathrm{S})$ is shown in Table 14.

Step 5. Normalizing the initial direct relation matrix

We apply the Eq. (7) to obtain the value of $\mathrm{K}$ and then the formula (8) to obtain the generalized direct relation matrix $\mathrm{X}$.

Calculation of each row:

$$
\left[\begin{array}{lll}
\text { Row } 1 & 1.86 \\
\text { Row } 2 & 1.76 \\
\text { Row } 3 & 1.58 \\
\text { Row } 4 & 1.82 \\
\text { Row } 5 & 1.78 \\
\text { Row } 6 & 1.77 \\
\text { Row } 7 & 1.83
\end{array}\right] \text { Max }=1.86 \mathrm{k}=\frac{1}{1.86}
$$

The generalized direct relation matrix $\mathrm{X}$ is presented in Table 15 .

Step 6. Attaining the total relation matrix

This step is performed using the Matlab software. The total relation matrix is acquired using the formula (9) from the generalized direct relation matrix X. A total relation matrix (T) is obtained, where (I) denotes the identity matrix. The total relation matrix is presented in Table 16.

Step 7. Obtaining the sum of rows and columns

The sum of rows is denoted by (D), and the sum of columns is denoted by (R), using the formulas $(10,11)$.

Sum of rows and columns

$\begin{array}{llll}\text { Col 1 } & 8.2182 & \text { Row 1 } & 8.8362 \\ \text { Col 2 } & 8.3654 & \text { Row 2 } & 8.2445 \\ \text { Col 3 } & 8.2309 & \text { Row 3 } & 7.2893 \\ \text { Col 4 } & 7.3264 & \text { Row 4 } & 7.5221 \\ \text { Col 5 } & 6.6465 & \text { Row 5 } & 7.1926 \\ \text { Col 6 } & 7.5996 & \text { Row 6 } & 7.1994 \\ \text { Col 7 } & 7.3456 & \text { Row 7 } & 7.4485\end{array}$




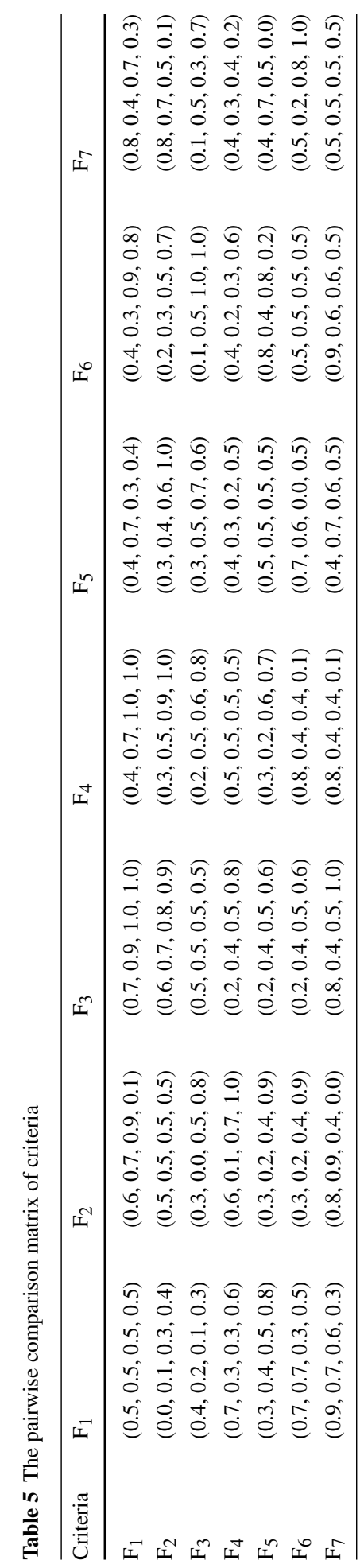




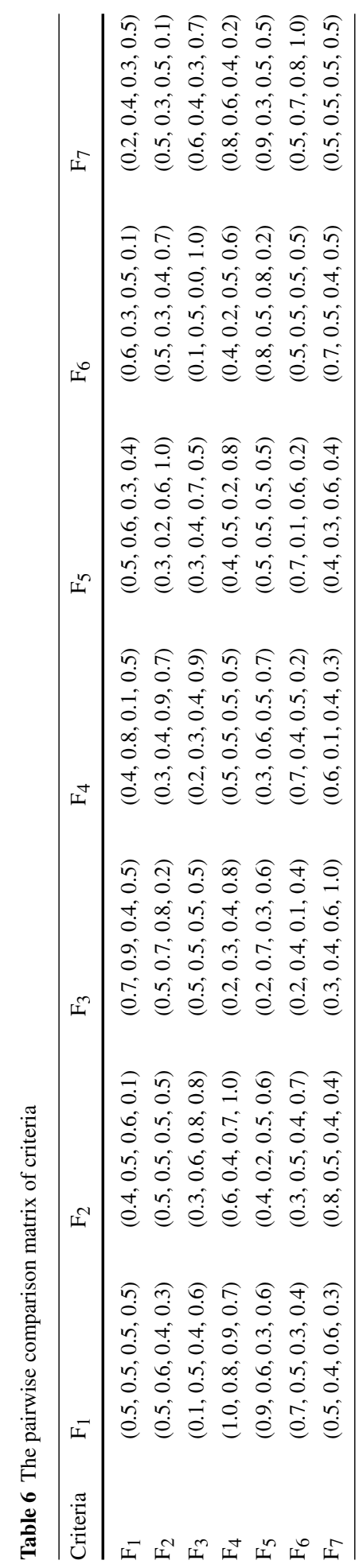




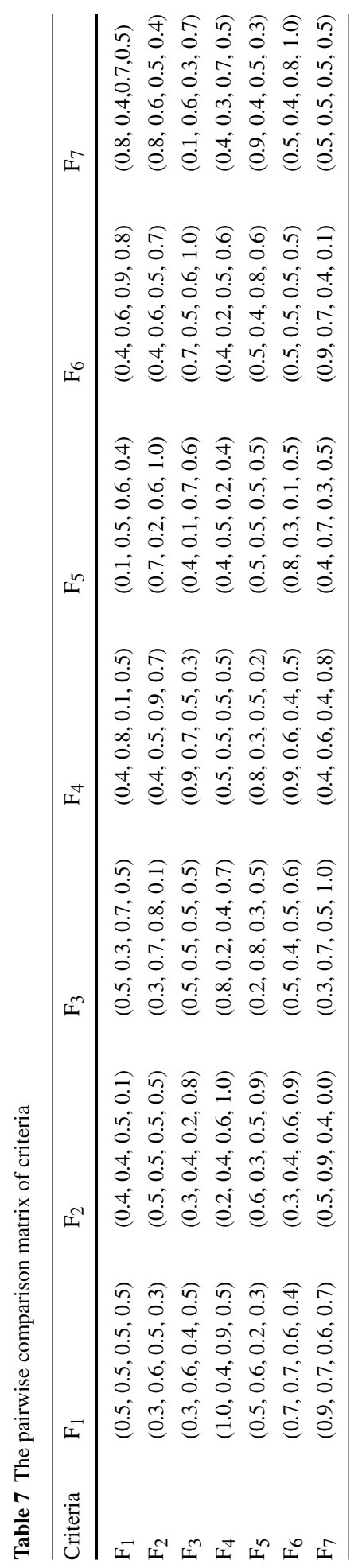




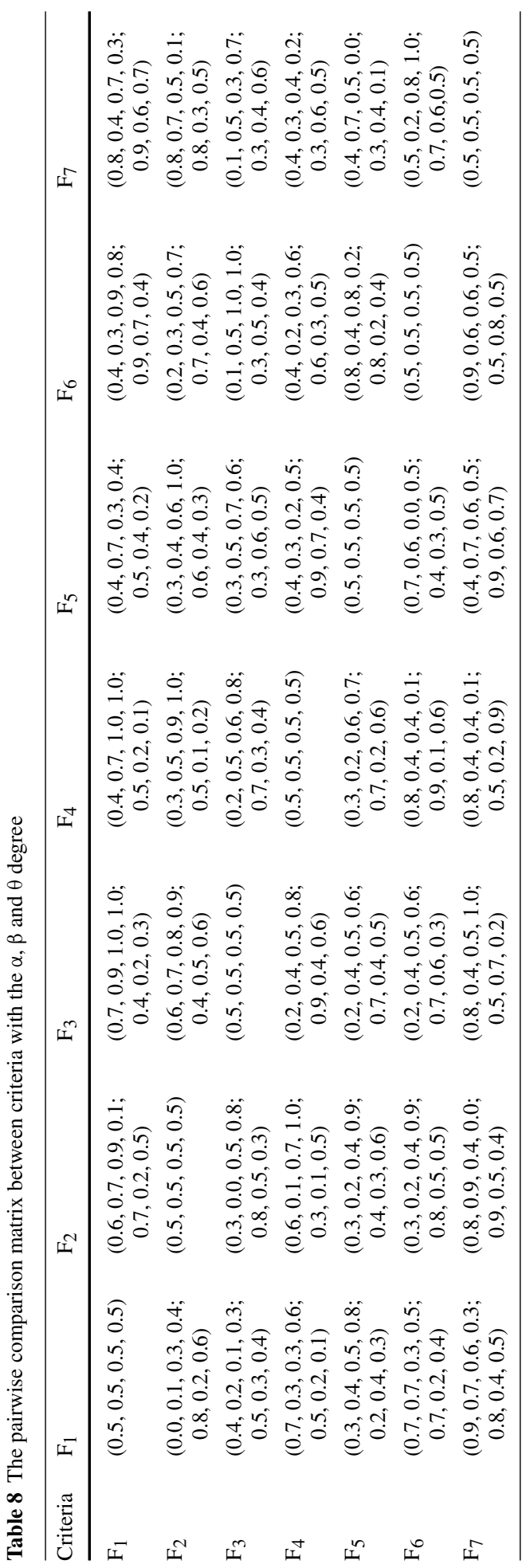




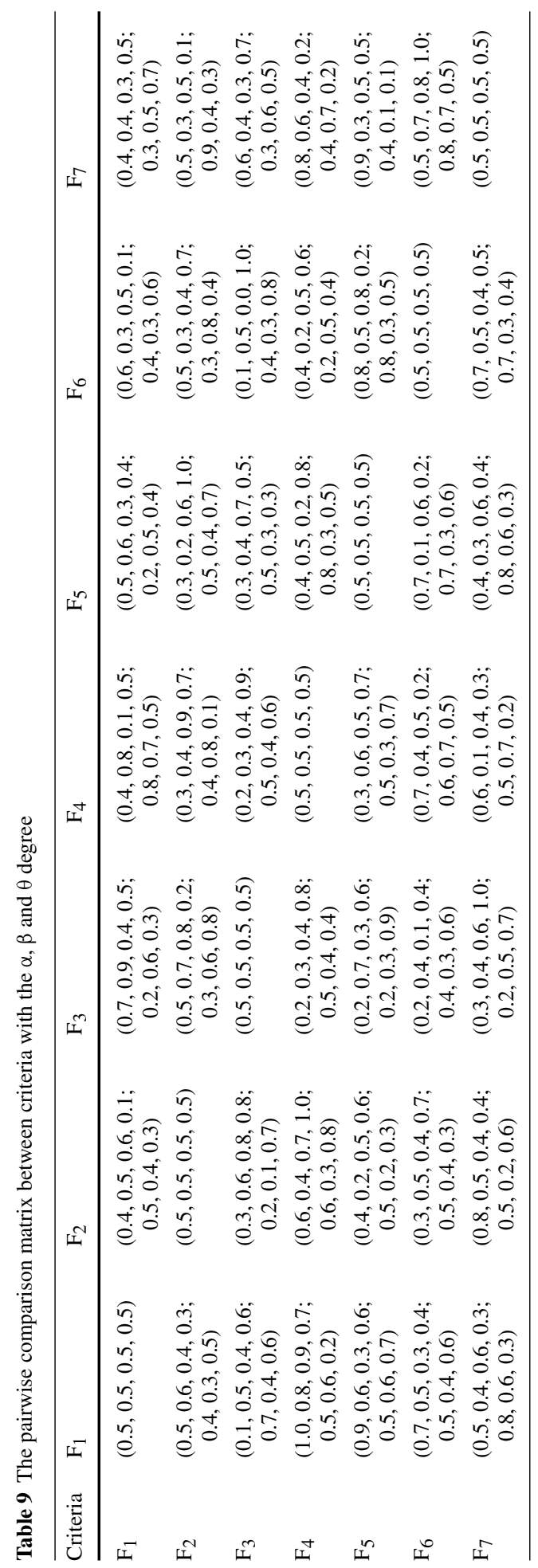




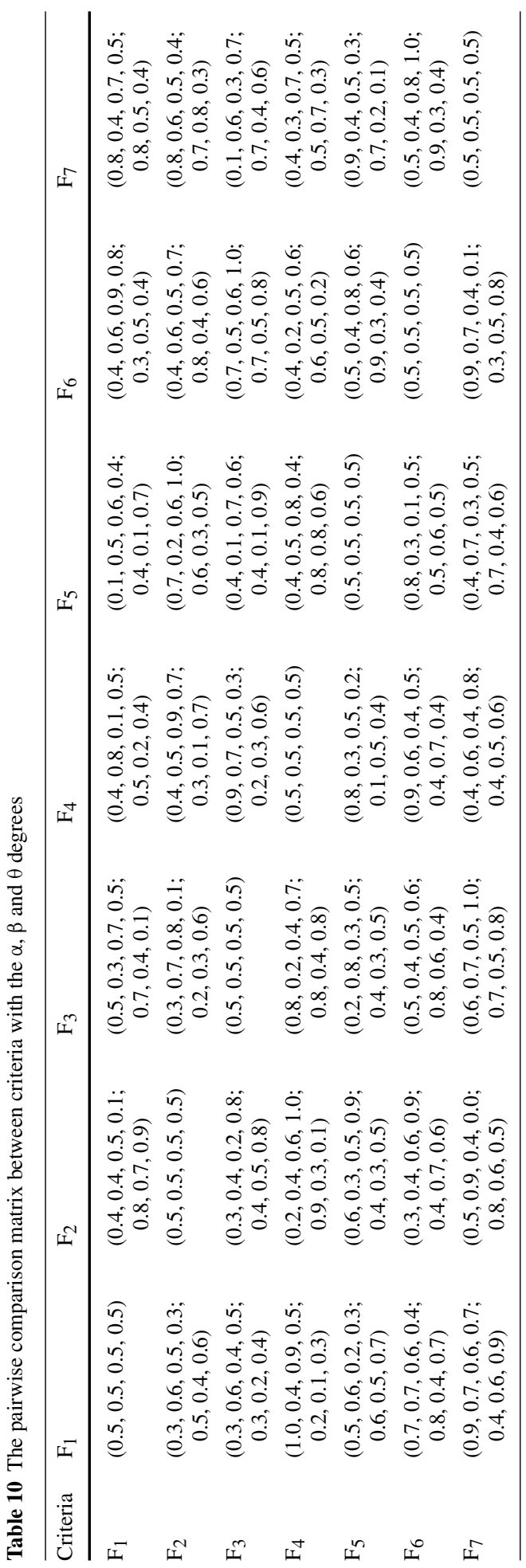


Table 11 The crisp values of comparison matrix

Table 12 The crisp values of comparison matrix

Table 13 The crisp values of comparison matrix

\begin{tabular}{llllllll}
\hline Criteria & $\mathrm{F}_{1}$ & $\mathrm{~F}_{2}$ & $\mathrm{~F}_{3}$ & $\mathrm{~F}_{4}$ & $\mathrm{~F}_{5}$ & $\mathrm{~F}_{6}$ & $\mathrm{~F}_{7}$ \\
\hline $\mathrm{F}_{1}$ & 0.5 & 0.288 & 0.428 & 0.426 & 0.214 & 0.270 & 0.220 \\
$\mathrm{~F}_{2}$ & 0.100 & 0.5 & 0.244 & 0.371 & 0.273 & 0.181 & 0.263 \\
$\mathrm{~F}_{3}$ & 0.113 & 0.200 & 0.5 & 0.263 & 0.158 & 0.228 & 0.130 \\
$\mathrm{~F}_{4}$ & 0.261 & 0.255 & 0.226 & 0.5 & 0.158 & 0.169 & 0.098 \\
$\mathrm{~F}_{5}$ & 0.188 & 0.169 & 0.191 & 0.214 & 0.5 & 0.303 & 0.181 \\
$\mathrm{~F}_{6}$ & 0.289 & 0.203 & 0.191 & 0.234 & 0.180 & 0.5 & 0.250 \\
$\mathrm{~F}_{7}$ & 0.296 & 0.263 & 0.270 & 0.149 & 0.220 & 0.195 & 0.5 \\
\hline
\end{tabular}

\begin{tabular}{llllllll}
\hline Criteria & $\mathrm{F}_{1}$ & $\mathrm{~F}_{2}$ & $\mathrm{~F}_{3}$ & $\mathrm{~F}_{4}$ & $\mathrm{~F}_{5}$ & $\mathrm{~F}_{6}$ & $\mathrm{~F}_{7}$ \\
\hline $\mathrm{F}_{1}$ & 0.5 & 0.180 & 0.203 & 0.180 & 0.146 & 0.141 & 0.110 \\
$\mathrm{~F}_{2}$ & 0.180 & 0.5 & 0.124 & 0.216 & 0.184 & 0.132 & 0.193 \\
$\mathrm{~F}_{3}$ & 0.170 & 0.219 & 0.5 & 0.169 & 0.226 & 0.130 & 0.150 \\
$\mathrm{~F}_{4}$ & 0.361 & 0.253 & 0.181 & 0.5 & 0.238 & 0.138 & 0.118 \\
$\mathrm{~F}_{5}$ & 0.180 & 0.213 & 0.113 & 0.197 & 0.5 & 0.288 & 0.303 \\
$\mathrm{~F}_{6}$ & 0.178 & 0.214 & 0.103 & 0.158 & 0.158 & 0.5 & 0.300 \\
$\mathrm{~F}_{7}$ & 0.214 & 0.223 & 0.144 & 0.140 & 0.202 & 0.263 & 0.5 \\
\hline
\end{tabular}

\begin{tabular}{llllllll}
\hline Criteria & $\mathrm{F}_{1}$ & $\mathrm{~F}_{2}$ & $\mathrm{~F}_{3}$ & $\mathrm{~F}_{4}$ & $\mathrm{~F}_{5}$ & $\mathrm{~F}_{6}$ & $\mathrm{~F}_{7}$ \\
\hline $\mathrm{F}_{1}$ & 0.5 & 0.105 & 0.275 & 0.214 & 0.160 & 0.236 & 0.285 \\
$\mathrm{~F}_{2}$ & 0.159 & 0.5 & 0.154 & 0.234 & 0.281 & 0.248 & 0.230 \\
$\mathrm{~F}_{3}$ & 0.191 & 0.117 & 0.5 & 0.195 & 0.158 & 0.245 & 0.181 \\
$\mathrm{~F}_{4}$ & 0.315 & 0.344 & 0.210 & 0.5 & 0.184 & 0.202 & 0.178 \\
$\mathrm{~F}_{5}$ & 0.140 & 0.230 & 0.180 & 0.135 & 0.5 & 0.316 & 0.315 \\
$\mathrm{~F}_{6}$ & 0.255 & 0.151 & 0.225 & 0.195 & 0.149 & 0.5 & 0.317 \\
$\mathrm{~F}_{7}$ & 0.163 & 0.191 & 0.245 & 0.179 & 0.202 & 0.131 & 0.5 \\
\hline
\end{tabular}

Table 14 The integration matrix

\begin{tabular}{llllllll}
\hline Criteria & $\mathrm{F}_{1}$ & $\mathrm{~F}_{2}$ & $\mathrm{~F}_{3}$ & $\mathrm{~F}_{4}$ & $\mathrm{~F}_{5}$ & $\mathrm{~F}_{6}$ & $\mathrm{~F}_{7}$ \\
\hline $\mathrm{F}_{1}$ & 0.5 & 0.191 & 0.302 & 0.273 & 0.173 & 0.216 & 0.205 \\
$\mathrm{~F}_{2}$ & 0.146 & 0.5 & 0.174 & 0.274 & 0.246 & 0.187 & 0.229 \\
$\mathrm{~F}_{3}$ & 0.158 & 0.179 & 0.5 & 0.209 & 0.181 & 0.201 & 0.154 \\
$\mathrm{~F}_{4}$ & 0.312 & 0.284 & 0.206 & 0.5 & 0.193 & 0.170 & 0.155 \\
$\mathrm{~F}_{5}$ & 0.169 & 0.204 & 0.161 & 0.182 & 0.5 & 0.302 & 0.266 \\
$\mathrm{~F}_{6}$ & 0.241 & 0.189 & 0.173 & 0.196 & 0.162 & 0.5 & 0.307 \\
$\mathrm{~F}_{7}$ & 0.224 & 0.226 & 0.220 & 0.156 & 0.208 & 0.208 & 0.5 \\
\hline
\end{tabular}


Table 15 Normalized matrix

\begin{tabular}{llllllll}
\hline Criteria & $\mathrm{F}_{1}$ & $\mathrm{~F}_{2}$ & $\mathrm{~F}_{3}$ & $\mathrm{~F}_{4}$ & $\mathrm{~F}_{5}$ & $\mathrm{~F}_{6}$ & $\mathrm{~F}_{7}$ \\
\hline $\mathrm{F}_{1}$ & 0.269 & 0.103 & 0.162 & 0.147 & 0.093 & 0.116 & 0.110 \\
$\mathrm{~F}_{2}$ & 0.079 & 0.269 & 0.094 & 0.147 & 0.132 & 0.101 & 0.123 \\
$\mathrm{~F}_{3}$ & 0.085 & 0.096 & 0.269 & 0.112 & 0.097 & 0.108 & 0.083 \\
$\mathrm{~F}_{4}$ & 0.168 & 0.153 & 0.111 & 0.145 & 0.104 & 0.091 & 0.083 \\
$\mathrm{~F}_{5}$ & 0.091 & 0.110 & 0.087 & 0.098 & 0.145 & 0.162 & 0.143 \\
$\mathrm{~F}_{6}$ & 0.130 & 0.102 & 0.093 & 0.105 & 0.087 & 0.147 & 0.165 \\
$\mathrm{~F}_{7}$ & 0.120 & 0.122 & 0.118 & 0.084 & 0.112 & 0.159 & 0.145 \\
\hline
\end{tabular}

Table 16 The total relation matrix

\begin{tabular}{llllllll}
\hline Criteria & $\mathrm{F}_{1}$ & $\mathrm{~F}_{2}$ & $\mathrm{~F}_{3}$ & $\mathrm{~F}_{4}$ & $\mathrm{~F}_{5}$ & $\mathrm{~F}_{6}$ & $\mathrm{~F}_{7}$ \\
\hline $\mathrm{F}_{1}$ & 1.4895 & 1.3104 & 1.3747 & 1.2188 & 1.0546 & 1.2165 & 1.1717 \\
$\mathrm{~F}_{2}$ & 1.1796 & 1.4323 & 1.1966 & 1.1458 & 1.0406 & 1.1287 & 1.1209 \\
$\mathrm{~F}_{3}$ & 1.0597 & 1.0920 & 1.2834 & 0.9900 & 0.8920 & 1.0158 & 0.9564 \\
$\mathrm{~F}_{4}$ & 1.1941 & 1.1955 & 1.1315 & 1.0582 & 0.9272 & 1.0271 & 0.9885 \\
$\mathrm{~F}_{5}$ & 1.0599 & 1.0988 & 1.0526 & 0.9618 & 0.9330 & 1.0672 & 1.0193 \\
$\mathrm{~F}_{6}$ & 1.1090 & 1.0905 & 1.0660 & 0.9717 & 0.8729 & 1.0503 & 1.0390 \\
$\mathrm{~F}_{7}$ & 1.1264 & 1.1459 & 1.1261 & 0.9801 & 0.9262 & 1.0940 & 1.0498 \\
\hline
\end{tabular}

$\begin{array}{ccc}\text { Row }+ \text { Column and } & \text { Row }- \text { Column } \\ 0 & \text { Row }+ \text { Col } & \text { Row }- \text { Col } \\ 1 & 17.0544 & -0.618 \\ 2 & 16.6099 & 0.1209 \\ 3 & 15.5202 & 0.9416 \\ 4 & 14.8485 & -0.1957 \\ 5 & 13.8391 & -0.5461 \\ 6 & 14.799 & 0.4002 \\ 7 & 14.7941 & -0.1029\end{array}$

Step 8. Drawing cause and effect diagram

The causal diagram is obtained by the horizontal axes, presented by $(\mathrm{D}+\mathrm{R})$, and the vertical axes $(\mathrm{D}-\mathrm{R})$, which is a degree of relation, as depicted in Fig. 6.

\subsection{Analyzing the evaluation criteria}

The final step is the analysis of collected data according to the causal diagram. This article integrates several questionnaires from expert interviews to find out the evaluation criteria and to calculate the average of each criterion. The research results determine the most important criterion. From this causal chart, according to the Neutrosophic DEMATEL Method, the importance of all criteria was established. According to experts' opinions, Quality (F3) had the greatest impact and Cost (F1) had the lesser impact on the selection of the company supplier. 


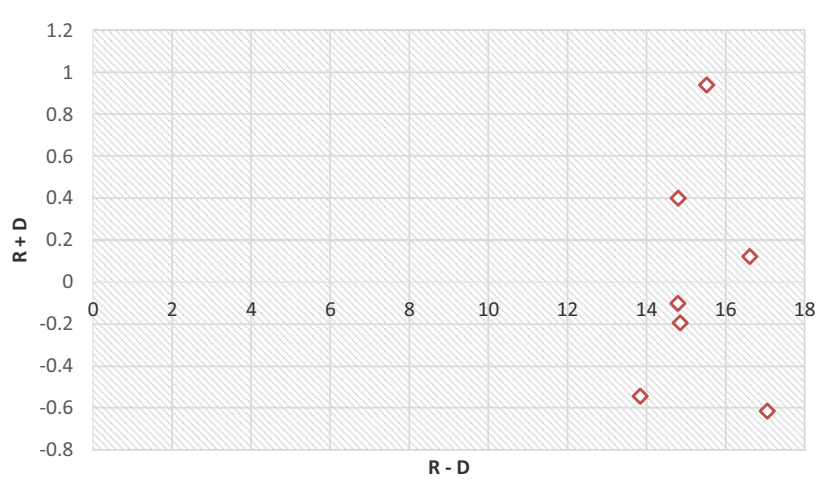

Fig. 6 The causal diagram for evaluation criteria

\section{Conclusions and recommendations}

This study presents the criteria selected by experts in the field of production and procurement in collective organizations, affecting the productivity and profitability of any organization. Potential supply chain management practices have been developed and performed using the Neutrosophic DEMATEL Method to select the best standards that have a greater impact on other criteria. The proposed approach succeeded in developing the DEMATEL Method by applying to it the Neutrosophic Set Theory, using a new scale from 0 to 1 and employing the maximum truth membership degree $(\alpha)$, the minimum indeterminacy membership degree $(\theta)$ and the minimum falsity membership degree $(\beta)$ of a single valued neutrosophic number. The opinions were collected from experts by interviews, and consequently analyzed using the Neutrosophic DEMATEL Method, by comparisons of each criterion, according to each individual expert, and their formulation of each value according to a single valued neutrosophic number. Finally, we extracted the most important criterion or feature that proved to be important for any organization in order to effectively choose its suppliers. However, this research contains some limitations and difficulties due to the fact that the multitude of standards and features require a large processing team and complex calculation.

\section{References}

1. Gharakhani D (2012) The evaluation of supplier selection criteria by fuzzy DEMATEL method. J Basic Appl Sci Res 2(4):3215-3224

2. Askarany D et al (2010) Supply chain management, activity-based costing and organisational factors. Int J Prod Econ 127(2):238-248

3. Gunasekaran A et al (2004) A framework for supply chain performance measurement. Int J Prod Econ 87(3):333-347

4. Arndt H (2004) Supply chain management. Springer, Berlin

5. Li X, Wang Q (2007) Coordination mechanisms of supply chain systems. Eur J Oper Res 179(1):1-16

6. Shyur H-J, Shih H-S (2006) A hybrid MCDM model for strategic vendor selection. Math Comput Model 44(7):749-761

7. Chang S-L et al (2007) Applying a direct multi-granularity linguistic and strategy-oriented aggregation approach on the assessment of supply performance. Eur J Oper Res 177(2):1013-1025

8. Dickson GW (1996) An analysis of vendor selection systems and decisions. J Purch 2(1):5-17

9. Gan J, Luo L (2017) Using DEMATEL and intuitionistic fuzzy sets to identify critical factors influencing the recycling rate of end-of-life vehicles in China. Sustainability 9(10):1873

10. Chan FT et al (2006) An AHP approach in benchmarking logistics performance of the postal industry. Benchmark Int J 13(6):636-661 
11. Zhang $\mathrm{C}$ et al (2017) Merger and acquisition target selection based on interval neutrosophic multigranulation rough sets over two universes. Symmetry 9(7):126

12. Smarandache F (1999) A unifying field in logics: neutrosophic logic. Neutrosophy, neutrosophic set, neutrosophic probability. American Research Press, Santa Fe

13. Smarandache F (2005) A unifying field in logics: neutrosophic logic. Neutrosophy, neutrosophic set, neutrosophic probability: neutrosophic logic. Neutrosophy, neutrosophic set, neutrosophic probability. Infinite study. American Research Press, Santa Fe

14. Abdel-Basset $\mathrm{M}$ et al (2018) Future generation computer systems. https://doi.org/10.1016/j.future.2018. 03.014

15. Abdel-Basset M, Mohamed M, Zhou Y, Hezam I (2017) Multi-criteria group decision making based onneutrosophic analytic hierarchy process. J Intell Fuzzy Syst 33(6):4055-4066

16. Hezam IM, Abdel-Baset M, Smarandache F (2015) Taylor series approximation to solve neutrosophic multiobjective programming problem. Neutrosophic Sets Syst 10:39-46

17. Smarandache F (2010) Neutrosophic set-a generalization of the intuitionistic fuzzy set. J Defense Resour Manag 1(1): 107

18. Chang B et al (2011) Fuzzy DEMATEL method for developing supplier selection criteria. Expert Syst Appl 38(3):1850-1858

19. Dey S et al (2012) Supplier selection: integrated theory using DEMATEL and quality function deployment methodology. Procedia Eng 38:3560-3565

20. Hsu C-W et al (2013) Using DEMATEL to develop a carbon management model of supplier selection in green supply chain management. J Clean Prod 56:164-172

21. Lin R-J (2013) Using fuzzy DEMATEL to evaluate the green supply chain management practices. J Clean Prod 40:32-39

22. Dalalah D et al (2011) A fuzzy multi-criteria decision making model for supplier selection. Expert Syst Appl 38(7):8384-8391

23. Govindan K et al (2015) Intuitionistic fuzzy based DEMATEL method for developing green practices and performances in a green supply chain. Expert Syst Appl 42(20):7207-7220

24. Florentin S (1998) Neutrosophy. neutrosophic probability, set, and logic. ProQuest information \& learning, Ann Arbor, Michigan, USA. http://fs.gallup.unm.edu/eBook-neutrosophics6.pdf (online edition). Accessed 17 Feb 2018

25. El-Hefenawy N, Metwally MA, Ahmed ZM, El-Henawy IM (2016) A review on the applications of neutrosophic sets. J Comput Theor Nanosci 13(1):936-944

26. Saaty TL, Vargas LG (2006) Decision making with the analytic network process. Springer, Berlin

27. Abdel-Baset M, Hezam IM, Smarandache F (2016) Neutrosophic goal programming. Neutrosophic Sets Syst 11:112-118

28. Mahdi IM, Riley MJ, Fereig SM, Alex AP (2002) A multi-criteria approach to contractor selection. Eng Constr Archit Manag 9(1):29-37

29. Atanassov KT (1986) Intuitionistic fuzzy sets. Fuzzy Sets Syst 20(1):87-96

30. Abdel-Basset $\mathrm{M}$ et al (2017) A novel group decision-making model based on triangular neutrosophic numbers. Soft Comput. https://doi.org/10.1007/s00500-017-2758-5

31. Abdel-Basset M et al (2017) Neutrosophic AHP-Delphi Group decision making model based on trapezoidal neutrosophic numbers. J Ambient Intell Humaniz Comput 1-17. https://doi.org/10.1007/s12652017-0548-7 